\title{
Interaction Criticism and Aesthetics
}

\author{
Jeffrey Bardzell \\ Indiana University \\ 919 E $10^{\text {th }}$ Street \#240 \\ Bloomington, IN 47405 \\ jbardzel@indiana.edu
}

\begin{abstract}
As HCI becomes more self-consciously implicated in culture, theories from cultural studies, in particular aesthetics and critical theory, are increasingly working their way into the field. However, the use of aesthetics and critical theory in HCI remains both marginal and uneven in quality. This paper explores the state of the art of aesthetics and critical theory in the field, before going on to explore the role of these cultural theories in the analysis and deployment of the twin anchors of interaction: the user and the artifact. In concludes with a proposed mapping of aesthetics and critical theory into interaction design, both as a practice and as a discipline.
\end{abstract}

\section{Author Keywords}

HCI, interaction design, interaction criticism, aesthetics, critical theory, cultural theory

\section{ACM Classification Keywords}

H5.m. Information interfaces and presentation (e.g., HCI): Miscellaneous.

\section{INTRODUCTION}

Accompanying HCI's move out of the workplace and into the home, the subway, the shopping mall, and virtual worlds is an increased awareness of HCI's participation in culture. This awareness is reflected in moves toward "experience" and "design," both of which have connotations that include but also clearly exceed the notions of performance, utility, and satisfaction that dominated the field in the past. This cultural sensibility goes beyond the avant-garde of experience design; it can be found in mainstream traditional HCI as well [e.g., 48].

Responding to HCI's rising cultural agenda are contributions in the fields of interaction aesthetics [e.g., 28,7,56,22] and criticism [e.g., 20,3,4,10,49,50,51]. Additionally, quite a bit of theorization on experience design [37,1], design practice [41], and research methods $[29,11]$ is built on the traditions of cultural theory that underlie contemporary aesthetics and criticism.

Permission to make digital or hard copies of all or part of this work for personal or classroom use is granted without fee provided that copies are not made or distributed for profit or commercial advantage and that copies bear this notice and the full citation on the first page. To copy otherwise, or republish, to post on servers or to redistribute to lists, requires prior specific permission and/or a fee.

CHI 2009, April 4-9, 2009, Boston, MA, USA.

Copyright 2009 ACM 978-1-60558-246-7/09/04 ...\$5.00
One challenge facing the HCI community is that aesthetics and criticism, as disciplines in their own right, are not part of our traditional disciplinary background. Briefly, HCI's mainstream disciplinary background includes its primary training inputs (e.g., HCI degree programs), disciplinary inputs (e.g., engineering, cognitive science, information systems), methodological predispositions (e.g., experiment, iterative prototyping, user research, ethnomethodology), and epistemological commitments (e.g., scientific rationalism and increasingly phenomenological human-centrism). This background evolved for legitimate historical reasons, and in many ways has served the field very well. While $\mathrm{HCI}$ as a discipline continues to expand, as does the significance of technology in society and culture, it is not going to - nor should it-radically upend its own history. That said, this disciplinary history has deep philosophical incompatibilities with that of aesthetics and critical theory.

The concepts and vocabulary of aesthetics and critical theory have much to offer HCI, because they emphasize qualities and issues that HCI is obviously concerned with in interaction: experience, symbolic density and cohesion, beauty, enlightenment, social justice, dialogism, identity and the self, form and meaning, taste and judgment, ideological encodings, interpretation/hermeneutics, and signifying structures, among many others. At the same time, these concepts were developed in, reflect, and continue to participate in very old traditions of theorizing. Removing them from these contexts and applying them to new disciplines carries with it risks of confusion and incoherence-and in doing so, may do more harm than good.

The goal of this paper is specifically to offer an introduction, for interaction designers, to aesthetic and critical theory as it applies to interaction. Doing so involves both laying out the recent historical agendas of aesthetics and critical theory, and situating them side-by-side with contemporary problems in $\mathrm{HCI}$ that stand to benefit from these lines of theorizing. These problems include, among others, tangible computing, interactive visualizations, mobile and ubiquitous computing, social and entertainment computing, evaluating user engagement, domestic and intimate computing, and designing creativity support tools. In setting out to achieve this goal, I seek to balance the need to be both accessible and to be substantial; this work is not intended to be "aesthetics and critical theory for dummies"; such content is already available elsewhere and anyway fails to ad- 
dress the particular concerns of HCI or its object of study: interaction. But a certain sensibility for aesthetics and critical theory is needed in HCI, not only to facilitate the introduction of aesthetic and cultural reasoning into HCI, but also to help provide grounds for this community to evaluate the quality and rigor of work in this area.

\section{PROBLEMS OF THE APPROPRIATION OF AESTHETICS AND CRITICAL THEORY IN HCI}

Many works that bring together $\mathrm{HCI}$ and aesthetics and critical theory have been published already, some of them quite influential. Without critiquing their contributions to $\mathrm{HCI}$ individually, it is possible to talk generally about the state of the art of aesthetic and critical concerns in HCI: the use of aesthetic and critical theory in HCI is uneven, still working itself through a liminal state of being neither true to its origins (in aesthetics, philosophy, and critical theory), nor particularly grounded in its destination (i.e., robustly integrated into the core theories and practices of our field).

Several common patterns have emerged in this process. One is when a single concept or idea it borrowed from aesthetics or critical theory and applied in the context of interaction design. Such an approach can have obvious benefits in the analytic utility it can bring to a design problem, but it also carries risks: the piecemeal appropriation of a single concept often strips it of much of its original analytic force, because concepts, especially in cultural theory, are sensible inasmuch as they participate or are deployed in networks of other concepts, issues, and historical events.

For example, the great film critic and theorist of the 1950s André Bazin [6] argued forcefully that camera shots with wide and deep focus were more ethical than close-ups and shots with shallow focus, because he considered them more accurately to reflect reality as it is, thereby putting the onus of its interpretation on the viewer, rather than on the director. Bazin's position entailed film practices attending to mise-en-scène, montage, and casting as well as complex philosophical positions on the relationships between viewer, film content, and director. This theorizing was also connected to contemporary filmmaking, as his work is most closely identified with Italian Neorealism, which it championed, and the later French New Wave, whose adherents considered Bazin a foundational thinker (though they reworked him).

Thus, appropriating any one technique, say, deep focus, from the rest of this context would rob it of much of its analytic force, since that technique is associated with and made meaningful by a series of films, a coherent theory of film, and an underlying theory of epistemology and ethics. Yet the reductive approaches of experimental science work best precisely when they can isolate on a single feature, control for it, and conduct a series of tests on it. This is not to suggest that there is no solution to this conflict, but merely to acknowledge that the conflict merits reflective action.

The Bazin example brings up another problem in the appropriation of aesthetics and critical theory into $\mathrm{HCI}$, and that is these deep conflicts are not treated with the seriousness they deserve. An example of this is [23], which offers a nuanced historical introduction to aesthetics before going on to define the aim of "aesthetic computing" as "the application of the theory and practice of art to the field of computing" (p. 6, italics in original). One example of this is "incorporating artistic methods in typically computingintensive activities, such as scientific visualization" (p.6). The concern I raise is not articulating these as goals, which seem provocative and interesting, but rather inasmuch as they gloss over just how difficult actually doing so really is.

The difficulty is not a mere matter of implementation. Rather, the philosophical underpinnings of artistic activity and scientific visualization are not obviously compatible. Another issue that artistic activity and aesthetics are not the same thing, as discussed below. The practical consequence is that we are pressured into one of the following scenarios: we do art well, and computing more dubiously; we do computing well, but the art is questionable; or we settle in and work through the deep difficulties of this activity with legitimate cross-domain expertise and reflective practice. Speaking generally (I am not now referring specifically about [23]), our field tends toward the second one, which is good computing and dubious art.

One way this tendency materializes is in the abundant frameworks offered in the field, which reduce culture and cultural theory to bullet lists. These guidelines may have a legitimate place in practice, and in many cases, they are presented as such. The problem is only when they are mistaken for a coherent theory and cited as authoritative resources on aesthetics, when they in fact make no such claim. An example of this is [7]'s 8-part framework for enabling designers to evaluate interface aesthetics. This framework is explicitly offered as a practitioner's guidelines, and it was tested and evaluated with graduate students and, on those terms, deemed successful. Considered philosophically, as a theory, the framework is incoherent, in that it integrates ideas from competing theories without any consideration that they seem to contradict each other.

In all of these cases, the pattern I am describing is not one of sloppy work; the works mentioned explicitly here, any many others like them, succeed on their own terms. Rather, the pattern is that inasmuch as aesthetics and critical theory appear in HCI, they tend to do so in pragmatic ways to solve particular problems. Harder to find (though by no means absent, e.g., [17]) is systematic, rigorous, expert integration aesthetic/critical traditions and HCI. A major reason for this state of affairs is that many of the people who are bringing aesthetics and critical theory into HCI have backgrounds in the sciences, including psychology, HCI, computer science, and so forth. Though they are serious scholars pursuing their work in earnest, they are not philosophers of art or critical theorists. On the flip side, experts of aesthetics and critical theory, to the extent that they even talk about computer technology at all, tend to do so in the context of new media (examples include [42,13,39,34]. 
They engage less with interaction design as a discipline, and so their work tends to have little influence in HCI.

If culture is incidental to HCI, then this situation, in which aesthetics/critical theory and HCI are not quite on the same page, is acceptable. But if we are to accept that $\mathrm{HCI}$ is a major cultural force in its own right, and if we are serious about experience design, then the lack of a pool of philosophically reasonable, coherent theories of interaction aesthetics and interaction criticism is a problem that needs to be addressed.

\section{AESTHETICS AND CRITICAL THEORY}

Throughout this paper two terms - aesthetics and critical theory - are used. To a casual observer, they may appear to be interchangeable, or perhaps one may appear to belong to the other. Be that as it may, the two terms historically represent two intellectual traditions that have evolved somewhat separately from one another, particularly in the twentieth century, and so to maximize their analytic utility for $\mathrm{HCI}$, they are worth keeping separate.

\section{The Discipline of Aesthetics}

As any recent monograph or handbook on aesthetics makes clear, aesthetics is the philosophy of art and beauty [e.g., $19,23,36,40]$. In particular, it is concerned with grounding, or philosophically legitimating, judgments about art and beauty. These judgments both involve normative judgments about what "good" art is, assertions and/or explanations of taste, as well as particular judgments about given works of art. Aesthetics has a long history, going back at least to the eighteenth century, where philosophers sought, among other things, to produce accounts of taste, then understood as a cognitive faculty, much like imagination or memory, which appreciated beauty. Along the way, key aesthetic terms were introduced and explored, such as the notion of art having special formal qualities that distinguish it from non-art, and the aesthetic as a special kind of experience or even an attitude.

As practiced today, the dominant strain of aesthetics owes much to the analytic philosophy of the twentieth century. This philosophical tradition emphasizes painstakingly careful logical reasoning that seeks to break down - and bring clarity to-otherwise fuzzy arguments and philosophical systems [54]. Working from definitions, contemporary aestheticians reconstruct arguments of prior aesthetic philosophers, relentlessly analyzing each argument, including its applicability to examples and its logical implications, to reveal its problems. For example, in his analysis of Collingwood's notion of art as the expression of emotion, [19] rearticulates an argument of Collingwood in the form of a logical proof, with sequentially numbered theses and an explanation of the logical transformation that produced each one (e.g., "assumption" and "deduction from 2 and 3").

Analytic aesthetics is not the only major strand of aesthetics. Another is hermeneutic aesthetics developed by Heidegger's student, Hans-Georg Gadamer [27], which marries the German tradition of aesthetics with phenomenology [33]. Alternatively, Richard Shusterman's Pragmatist Aesthetics [53] builds on the analytic tradition using the pragmatism of Dewey and Rorty to propose an experiencecentered aesthetics. Aesthetics is, in all of its variants, a philosophical discipline. It contains within it a body of key concepts (e.g., taste, art, craft, institution, emotional expression, beauty, aesthetic experience, significant form, artworld), and this body of concepts has a history. Aesthetic philosophers have mastered this history, and they use careful philosophical reasoning critique the limits of and revise these concepts and their relationships to the ever-changing world of art.

One might contrast such a notion of aesthetics with the understanding of aesthetics implicit in, for example, Nielsen's heuristics [44], which is based on a notion that the aesthetics of interaction merely inhere in the decorative elements that adorn an interface. Neilsen's position is rejected in a recent Interactions article [28] as superficial. Yet in the same article, the author offers in its place a notion of aesthetics driven by four qualities, two of which are "utility" and "performance." These qualities bear a non-trivial resemblance the traditional values of $\mathrm{HCI}$ and come across more as commonsense than as a philosophically justifiable framework. Whether this new notion is useful for practitioners is beside the point; it is itself a debatable philosophical position that in aesthetics would be subjected to rigorous scrutiny.

If HCI wants to engage with aesthetics, then rather than making up its own frameworks, it should work with the best aesthetics has to offer. Such an integration might work from the analytic aesthetic tradition, whose shared history with the philosophy of science would seem to make it compatible with empiricist and positivist approaches to interaction. Alternatively, the integration may come via Gadamer and other hermeneutic philosophers of aesthetics, including Ricoeur and Arendt, whose phenomenological orientation would seem to provide a promising entry into humancentered aesthetic interaction. Likewise for Shusterman, whose pragmaticism also seems to speak to important trends in $\mathrm{HCI}$, in particular the marriage of pragmatism and experience design. Regardless, HCI should not rest on the very same kinds of commonsense or ad hoc notions that aesthetics has spent generations dismantling.

\section{The Discipline of Critical Theory}

Critical theory is an umbrella term for the theories developed in and for (what would eventually become) cultural studies. (Critical theory is also used to describe the Marxist approaches of the Frankfurt School, but the term is used throughout this paper in the general, not Frankfurt, sense.) It includes literary, political, linguistic, psychoanalytic, and film theories, among others, and has taken any aspect of culture as its object of study, from modernist art to sumo wrestling. 
Cultural criticism is not primarily about taste, or identifying which are good books or movies and which are bad ones. Instead, criticism is typically aligned with continental philosophy, the main rival of analytic philosophy described earlier under aesthetics. Continental philosophy is situated in the radical skepticism of Nietzsche and continues in the twentieth century through the phenomenology of Heidegger; existentialism and Sartres; poststructuralism, Foucault, and Derrida; several generations of feminism from de Beauvoir to Paglia; and the postmodernism of Lyotard and Baudrillard, among others.

Continental philosophers reject the notion that humans have objective access to the external world. Knowledge, therefore, can never be a representation of an external reality (since we have no access to it); knowledge instead is a construction, created under particular circumstances in a time and place. The implications of this theory are far-reaching, because they imply that knowledge is situated in personal, social, conceptual, political, and other dynamics. Neither objective nor universal, knowledge is treated as subjective and, above all, contingent. Its contingency opens it up to critique and the possibility of change.

As a part of its skepticism, continental philosophy often challenges the legitimacy of science and scientific ways of knowing. This creates an interesting tension in HCI. On the one hand, its skeptical stance toward science understandably alienates many in HCI, as evidenced on a recent panel on the state of the field in HCI [8], where a recurring theme was the need to protect the "science of design" from threatening postmodern encroachments. At the same time, one of the key underpinnings of user research is that the "truth" of an information system - whatever that might mean - is irrelevant; it is the users' understanding of the system that matters. "User-centered design" is fundamentally phenomenological, because it is grounded in, that is, its source of data originates in, the understandings, interpretations, and everyday practices of users, rather than the external world as such.

Critical theorists develop ideas articulated in continental philosophy in cultural works to explore the constructedness of knowledge. This exploration takes place from a number of angles-linguistic, ideological, gender -based, institutional, environmental, etc. The inputs into knowledge constructs are understood as diverse, complex, intentional, subconscious, implicit, tacit, irrational, genealogically layered, ideological, linguistic, and ritually structured-all at once. Indeed, these conditions are understood to be mutually reinforcing and constitutive. Where analytically inclined aestheticians seek to break down complex arguments into constituent parts, in order to subject them to investigation, critical theorists tend toward synthesis.

The history of feminist theory exemplifies these characteristics. As [38] relates, feminism has gone through a number of phases, e.g., liberal feminism, radical feminism, multicultural feminism, and postmodern feminism, among oth- ers. The phases differ largely in the way feminist concerns interact with other intellectual movements, such as liberal humanism, poststructuralism, and Marxism; these interactions constitute feminism, including its working conceptualization of "woman"; its intellectual strategies, alliances, and units of analysis; and its ethical positions and political goals. Womanhood itself is not treated as a biological fact, so much as a knowledge-construct deserving of critical examination, hence Simone de Beauvoir's famous statement, "One is not born, but rather becomes, a woman" [18]. Feminisms variously critique images of women in media, policies and laws pertaining to women, gendered institutional practices, scientific discourses on/about women, and other places where/mechanisms through which woman is constituted in and by knowledge. By exposing how women become enmeshed in these discourses, feminists introduce opportunities for social action.

Perhaps the main threat in the appropriation of critical theory in HCI is the piecemeal use of its key concepts. Taken out of context, critical concepts lose much of their original explanatory power, and when they are attached to otherwise rationalistic approaches, they can be even further diminished, impoverished, and trivialized. Thus, when critical theory seems most robust to those in the humanities, it may appear at its most frustratingly dense, fuzzy, and even downright absurd to scientists and practitioners; conversely, when it appears operationalizable and actually useful to scientists and practitioners, it can appear mechanistic and trite to critical theorists. The bipolar attitudes to postmodernism bears witness to this. Again, none of this suggests that the gulf cannot be bridged; it mainly calls attention to the depth of that challenge.

\section{Disciplinary Goals Shared by Aesthetics and Critical Theory}

If critical theory and aesthetics are different traditions with different key thinkers, critical strategies, and notions of legitimacy, they nonetheless share some commonalities. One of them is a rejection of the notion that art and culture "is all subjective," which is a counterproductive cop-out. Both aesthetics and critical theory are oriented to development of practices of expert judgment, specifically to make possible judgments about art and culture. To be sure, their notions of judgment - and its evaluation-differ. In analytic aesthetics, judgment is grounded in relentless logical reasoning. In critical theory, judgment is cultivated through the deconstruction of knowledge as it appears in - and produces-culture.

Aiding in the development of a discipline of expert judgment is the elaboration of difficult to define or operationalize concepts, such as catharsis, the sublime, ideology, simulacra, affective stylistics, embodied subjectivity, ecocriticism, and postcolonial identity. These concepts themselves participate in networks of other concepts, historical events, disciplinary traditions, artistic traditions, institutions of power, and popular media, and experts in critical theory and aesthetics, in making cultural judgments, confront those 
networks head-on. Judgment is a key feature of professionalism in other design fields [43], and is increasingly being championed in HCI [e.g., 32]. Yet judgment is still treated with skepticism by many in HCI, who do not understand how it differs from mere opinion. Expert judgment differs from opinion inasmuch as it engages in disciplines of judgment, such as aesthetics and critical theory, that offer the intellectual tools to develop rational arguments about cultural phenomena that are difficult or impossible to measure or evaluate scientifically.

\section{CRITICAL APPROACHES TO INTERACTION}

Though it has its origins in a number of fields, such as linguistics, philosophy, social theory, and literary theory, critical theory is commonly treated as meta-theory for the study of culture through the arts. One can, for example, take a Marxist, deconstructive, feminist, or semiotic approach to works of literature, film, opera, painting, dance, or mass culture (e.g., advertising and TV sitcoms). In this sense, the theory in cultural theory differs from that of scientific theory. It is not discrete, verifiable statement that models or predicts a phenomenon, nor is it an expression of a law of non-observables, as in Carnap's [16] examples regarding the behaviors of electrons.

Cultural theory, in contrast, might be fairly described as embodying complex and insoluble philosophical positions, which entail (ontological) assumptions about the nature of reality, epistemological possibilities and constraints, methodological strategies, and ethical stances all at once. Thus a Marxist critic stresses the emancipation of the masses, by simultaneously exposing the hidden conservatism of existing discourses, such as novels or films, and dialectically opening them to the possibility of revolutionary change. Within this theory are a number of theoretical conceptsdialectic, base, superstructure, ideology, fetish, alienation, materialism, etc. - that facilitate the Marxist's critical activity. These theories are not verifiable or predictive in the same way that a theory about the behavior of molecules is. Rather, they are intellectual tools to support the interpretative and activist activities of the critic. Other cultural theories, such as structuralist semiotics, may have only an indirect orientation toward social activism, but they still entail epistemological possibilities and limitations, a view of the nature of the world, a set of methods, and ethical positions (obviously, an indirect relationship toward activism is itself an ethical position).

Does interaction belong in the list of cultural phenomena, alongside film, novels, operas, and TV, or put another way, is interaction the kind of thing that a cultural studies approach could critique? From the standpoint of cultural criticism, the answer is obviously yes. From the standpoint of $\mathrm{HCI}$, which is oriented toward the design and evaluation of real-world interaction design as opposed to cultural critique for refined judgment or broader social activism, the answer is perhaps more nuanced. Two issues are at stake: one is the comparability of interaction to other cultural forms, and the other is the orientation not towards social activism, but rather towards immediate, concrete, and situated interaction design problems.

In an earlier paper [4], we argued that criticism historically examines cultural artifacts from one, or a combination, of the categories in Table 1.

\begin{tabular}{|l|l|l|}
\hline Category & Examples & Approaches \\
\hline Creator & $\begin{array}{l}\text { Author, designer, } \\
\text { composer, painter }\end{array}$ & $\begin{array}{l}\text { Biographic, psycho- } \\
\text { analytic }\end{array}$ \\
\hline Artifact & Text, film, product & $\begin{array}{l}\text { Semiotics, rhetoric, } \\
\text { deconstruction }\end{array}$ \\
\hline Consumer & $\begin{array}{l}\text { Reader, viewer, } \\
\text { user }\end{array}$ & $\begin{array}{l}\text { Reception theory, eth- } \\
\text { nography }\end{array}$ \\
\hline $\begin{array}{l}\text { Social con- } \\
\text { text }\end{array}$ & $\begin{array}{l}\text { Community, sub- } \\
\text { culture, workplace }\end{array}$ & $\begin{array}{l}\text { Marxism, feminism, } \\
\text { queer theory }\end{array}$ \\
\hline
\end{tabular}

Table 1: Traditional categories of criticism

All of these appear to have fairly straightforward potential for applicability to interaction. But two of them seem especially important for interaction, since together they constitute it: the artifact and the consumer (or "user," as this is known in interaction design).

\section{The Artifact-User and Interaction}

In our everyday understanding of traditional art and design, the artifact and the user are obviously separate entities: there is the novel and its reader, the film and its viewer, the building and its resident, the sonata and its listener. Challenging this everyday categorization scheme, critical theory in the latter part of the twentieth century critiqued this separation as missing the most important aspect of reading: its hermeneutic reception by the reader. (Note: "reading" often is used figuratively for "engaged interpretation and experience of a cultural artifact," regardless of whether it is literally a text.) As theories of the phenomenology of reading became more sophisticated (especially in the domains of reception theory and late semiotics), a position emerged that suggested that a text only exists when it is being read by a reader, and that no two readings (even of the same text by the same reader) are ever the same (e.g., $[5,21,55])$. The text itself, in this theory, exists in potential, and it is actualized only in the act of reading. As [39] pointed out over a decade ago, the applicability of the notion that a text is its performance by a reader, rather than the marks printed on paper, is much more obvious in the case of interaction. As we interact with interaction designs, we create its sequences, its juxtapositions, its meaning and significance.

Imagining interaction design artifacts as separate from users, and vice-versa, is certainly possible, but in $\mathrm{HCI}$, we try to blur the distinction and have done so for a long time. Norman's [45] framework, which explicates the relationships among the design model, the system image, and the user model is oriented toward harmonizing, not differentiating among, the three. HCI has developed techniques to facilitate this harmonization by elaborating conceptual 
models of systems, predictive performance models (e.g., GOMS), user mental models, and human cognitive models and conscientiously designing systems to be compatible with all of the above. Other movements, such as participatory design, albeit in different ways, have also worked toward this goal.

A textbook example (literally: [52]) is VisiCalc, the original electronic spreadsheet. Building on familiar metaphors and practices (e.g., the paper ledger, accounting), taking into account professional needs (e.g., economic forecasting), and human limitations (e.g., slow and error-prone calculation), VisiCalc harmonized human cognition and computational capabilities in an elegantly simple, yet professionally potent, conceptual model. The fundamentals of its design still stand at the core of Microsoft Excel over 20 years later.

As a strategy of interaction design, the spirit of VisiCalc lives today in the field of scientific visualization. For example, in [46] a visualization tool, SocialAction, was developed to support exploratory social network analysis, whose data sets are so large that researchers struggle to gain insight from them efficiently. Like VisiCalc before it, Socia1Action is built on familiar metaphors (e.g., network diagrams and GUI widgets, such as sliders), professional needs (e.g., analyzing complex large group dynamics), and human abilities and limitations (e.g., the human preference to analyze "complex data with images rather than numbers"). SocialAction harmonizes relevant cognitive models and needs of its users to computational capabilities (as augmented by years worth of innovation in statistical algorithms) to create an elegant solution usable even by those not familiar with social network analysis (SNA).

The success of tools such as these may obfuscate that they implicitly take critical positions, which presumably are quite appropriate in the immediate contexts of these applications, but which are nonetheless philosophically contingent. That is, the "transparent" harmonization of user and interface constitutes what would, in criticism, be called a theory of hermeneutics. In this tacit theory, an interface solves a well defined problem in a coherent, cognitively supportive, and explicit way. For if SocialAction enables users to interact with data in exploratory and ways unpredictable to the designer, nonetheless, SocialAction situates users, data, and itself in a stable relationship to each other Put another way, SocialAction supports emergent analysis, but it does not support emergent use.

This theory of interface hermeneutics has been challenged by [50]. They observe that lack of precise clarity of understanding between designer and user up-front is treated in HCI a priori as a problem to be solved. However, [50] note that many technologies are successful not in spite of, but because of this lack of clarity: they offer the example of SMS messaging, which is used in very different ways by different populations and over time (e.g., scheduling business meetings, teens with mobiles, and more recently mixed reality communications between mobile phones and residents of virtual worlds).

Senger \& Gaver's critique should not be taken to suggest that applications such as SocialAction are wrongheaded. Rather, the critique is of the often unexamined normative assumptions in $\mathrm{HCI}$ in general (including in its core textbooks) about the way humans and computers should interact. If the transparency model described earlier is useful for massive data visualization-based analysis, perhaps some other model could become the foundation of an entirely new technology or user research methodology.

This dynamic is visible in user engagement research, where conflicting models of affect - the information processing model of [47] versus the cultural interactional model of [12] - suggest substantially different approaches to the measurement/interpretation of user engagement. At the core of the debate is a philosophical dispute, but in practice the debate itself is pushing innovation in emotional computing. In other words, regardless of how or even whether the philosophical debate over affect is resolved, it has sustained a diverse and rich research agenda.

In short, a disciplined critique of the nature of the unity of artifact-user in interaction promises to stimulate innovation, particularly in the more obviously cultural problem spaces of HCI: entertainment, domestic, sustainable, affective, and aesthetic computing. Likewise, it promises to help us think deeply about new paradigms of computing, such as mixed reality, ambient, virtual world, tangible, and ubiquitous computing. It is not, therefore, fundamentally oriented towards claiming that such-and-such a tool is good, or could have been improved, as if it were a movie review. Rather, critical approaches are about exposing and exploring alternative assumptions about the key relationships in our field - the user, the design, interaction, the business or home context, and quality of life now and in the future. In this spirit, it is worth exploring the ways "the user" and "the design artifact" are (or could be) constructed and the implications of these constructions for interaction design.

\section{Constructing the User}

Commonsensically, the "user" is an unproblematic category: it is the flesh-and-blood person who uses a given piece of software. But this commonsense notion obfuscates some fundamental changes in the conceptualization of the user in the history of HCI. These changes are more or less familiar to everyone in the field: early notions of the user as a cog in the machine, a human factor; the user as a cognitive model; the user as an actor situated in a context; the user as a symbolically dense identity participating in a cultural context.

These constructs matter, because they shape research and development at every level of the system, from user research approaches (e.g., lab based usability tests, contextual inquiry, ethnomethodology, ethnography, cultural probes) to iterative design and evaluation strategies. 
The category of the "user" in HCI is both an empirical phenomenon waiting to be studied and a speculative construct. On the face of it, this statement appears to be contradictory, but it rests on a logical distinction between extensional and intensional reference. Extensional reference is the set of things in extramental reality to which a word refers; in this sense, the user is any actual flesh-and-blood person who uses the system. Intensional reference is the understanding called to mind, which is always an abstraction. Intensionally, the user usually refers to the kind of person that we consider to be an appropriate or felicitous user of a system. Thus, a child mashing on a parent's keyboard can extensionally be a "user" of a spreadsheet, inasmuch as there is actually a person present who is actually interacting with the software. But no designer would or should consider that child a "user" in the intensional sense. In conducting empirical research, we engage in both extensional and intensional senses of reference; we find actual people (extensional) to serve as a sample to a population (intensional). The risk is that the comforting presence of real people as users can obfuscate subtle distinctions and reasoning about those people inasmuch as they participate in the artifact-user construct that is interaction.

Let us consider online identity as an example. We have a comfortable mental model of the "real" person who is sitting in front of a computer, and this user has a representation online. This representation is evaluated in terms of how truthfully it represents the real person at the keyboard. Now, this conceptualization I have just described, though widespread, works far better in some HCI problem domains than in others.

In cybersecurity, the representational model is vitally important. When a user interacts with an online banking application, it is essential that the representation of the user to the bank is true; equally important, the representation of the bank to the user must be true. If either of these is untrue, the resulting situation is fraud; either someone is impersonating a user and can steal her or his money, or, as in the case of phishing, someone is impersonating a bank and can potentially steal people's online identities, leading to fraud. This conceptualization of the user gives rise to the problem of trust - evaluating it, guaranteeing it, and signifying itwhich is central to cybersecurity and HCI.

Is this representational model appropriate in virtual worlds, such as World of Warcraft or Second Life? This would entail expectations that avatars would truly correspond to its user, a desire raised often by business users of virtual worlds. To approach an answer, one must consider its implications. The representational model of identity puts "reality" strictly outside of the computer. In banking applications, this is where the reality should be. But in "virtual reality," it seems self-defeating to situate reality outside of the computer; surely there is some reality in virtual reality, at least as it is experienced by its users. If that is the case, it suggests rather than a representational model consisting of two units-i.e., digital representation and real self - that a hybrid model-i.e., the user online - makes more sense. This conceptual shift has a range of implications. It suggests that deeply personal issues - tastes, values, prejudices, intimate expressions - themselves become hybridized. In this view, activity, including friendship and collaborative play, in virtual worlds is not purely imaginary, but neither is it fully real.

To negotiate the symbolic complexity of this hybrid identity, we propose in [2] the appropriation of the French philosopher-sociologist Michel Foucault's [25] own appropriation of the ancient Stoic notion of askessis, which in Foucault's hands becomes an analysis of selfhood as an ongoing work of art made possible through "technologies of the self." The latter include a range of related ideas, including sexuality, the desire for knowledge, one's own engagement with culturally available identity stylistics, and so on.

One of these, for example, has an obvious implementation in virtual worlds: virtual world identity stylistics are made possible through avatar design interfaces, which can, especially in the case of Second Life, easily be seen in the category of creativity support tools. Indeed, Second Life's avatar design interface has numerous similarities with programs such as Photoshop and Flash [3]. It is also enormously productive, as witnessed by the proliferation of Second Life fashion blogs. Avatar visual design is also significant, as it is an important aspect of online professionalism, according to IBM's Virtual World Guidelines.

The design of next generation virtual world communication and sociability tools hinges on these issues. A critical examination of the relevant constructions of the user-as implied in interfaces, as developed by users, as appropriated by corporate uses of virtual worlds - is essential to the design of technologies that support the profoundly cultural uses of these worlds. These constructions are a moving target; the notion of virtual identity is dynamic, a living part of virtual worlds, and, frankly, part of what makes them so compelling.

Finally, it is important to note that both of the constructions of user summarized here - the representational model versus the subjectivity model - apply to virtual worlds at different levels. Fraud can and does occur in virtual worlds, and so at the level of authentication, where real-life credit cards, permissions, and other account attributes are at stake, the representational model of identity is relevant, as are attendant issues of trust, phishing, and so on. Thus, the "user" half of the artifact-user unit of analysis advocated here turns out to be a complex and evolving set of relationships between actual users (extensionally referenced) and user constructs (intensionally referenced). This characterization is robust enough to handle the sheer diversity of user research methods - and implied epistemologies - of our field, from experimental studies to cultural probes.

\section{Constructing the Artifact}

As with much of the previous argument, one can distinguish between commonsense notions of the artifact as given, as 
"just there," and more critical understandings of the artifact as a series of constructions among designers and different users and even uses. Design artifacts are constructed in a number of different ways. These include notions of the artifact as a tool, a work of rhetoric, and a participant in ecology. Each of these conceptualizations has deep implications not only for the functional design of software, but also for its ethical implications - for designers and users alike.

The notion that software is a tool is quite common in HCI, and, as always, it has a number of implications. In the mainstream of creativity support software in HCI, as represented in the Creativity and Cognition conference, $\mathrm{CHI}$, and even the NSF-sponsored CreativeIT program, creativity support software products are "tools." Creative agency is situated in the creative professional, who merely uses creativity support software to accomplish creative activity.

As described in [3], this rationalist notion of creative agency leads to practical design decisions that end up imposing a theory of creativity on the user. It does so by structuring the interface as a series of tasks, articulated in a professional vocabulary, without any content (i.e., the Photoshop, Flash, or Final Cut Pro workspace starts empty), which the user is expected to provide. These particular interface features are often derived or adapted from other GUI interface metaphors and interaction design strategies (e.g., task analysis and design). This style of interface, in turn, separates source materials (e.g., a photograph) from their algorithmic manipulation in software (e.g., running a Gaussian Blur filter). "Creativity" in this software is actually an activity of compositing [42]. It also treats different arts-music, photographic image editing, line drawing, animation, video, multimedia presentations - as having fundamentally similar approaches.

All of this is a transformation, rather than an extension, of art as it historically been produced. This transformation may not be intrinsically bad, but it does leave open questions of whether creativity is being "supported" or "imposed," and if the latter, what its professional (e.g., job skills, education) and cultural significance is. Emergent uses of video games as platforms for creating videos, called machinima, appear to reject the dominant paradigm of creativity support tools because the games provide precomposited content and intuitive interfaces, rather than blank screens and import interfaces and manipulation tools mediated by professional vocabulary and concepts. Why the rejection of the creativity support paradigm among amateur creatives? And what are the implications for design?

Another conceptualization of the artifact is that it is a work of persuasion [24] or rhetoric [15]. For [24], technology can be used in persuasive ways (which suggests that some technologies are not persuasive). For [15], because technology always could have been designed another way, every design becomes an argument for itself, in particular "issues of practical action," which are modeled and projected by the design. For both, persuasion/rhetoric is not merely transi- tive from the intentions of the designer to its effects on the user; in some sense, persuasion is projected by the interface itself.

Combining the past two points, the persuasive/rhetorical artifact argument suggests that an inanimate object-a design - is conditioning everyday, practical living. This claim has obvious ethical implications, which both [24] and [15] explicitly consider. Note that ethics is all but irrelevant if a design is just a tool, because ethical agency is situated squarely in the user. But if designs persuade people, or reshape everyday life, they can in that limited sense be understood to exercise agency and have an ethical dimension.

If individual artifacts can exercise a certain form of agency, what is the cumulative effect of our living in an artificial world, populated by persuasive designs created by millions of different designers in different fields, from toothbrushes and business cards, to creativity support tools and flight simulators? This argument is explored in [57,26], who use the phrase "ontological design" to account for the cumulative non-human agency of designs in the artificial world on human life in general, arguing that as people design artifacts, so the artifacts design us. Much of the early emphasis on sustainability in HCI emerged from this thinking, as has been carried forward by others. With sustainability, a deep philosophical position, which in [57,26] began as a phenomenological reconsideration of design, focused on a particular issue - sustainability - which led to the construction of frameworks [9], empirical research [33], and design strategies [14].

Critical examinations of artifacts create opportunities to develop new ideas, follow through with subtle yet potentially life-altering implications, and initiate new research agendas.

\section{CONCLUSION}

The idea that knowledge is constructed and contingent, rather than objective and representational, is not merely a cause for postmodern hand-wringing. It can contribute directly to design, by opening up strategies of disciplined speculative reasoning. Because all design is oriented to create change, not merely discover what-is, speculative reasoning ought to be a core skill of the discipline, as it is in traditional design. The problem is distinguishing speculative reasoning from mere opinion or pure fantasy. To ensure the groundedness and relevance of designerly speculation, we need the union of, not competition of, scientific and critical ways of knowing. Our problem space-humancomputer interaction - is an elusive object of study. We cannot see it directly, even less so during the design conceptualization phases. But empirical science, including all forms of user research, can help us understand the phenomenology of interaction, an argument that the field appears to have increasingly accepted after [58]. In a complementary way, critical approaches help us think deeply about how we as designers, and how users as users, construct knowledge about artifacts and users, which stimu- 
lates innovation and helps HCI engage its cultural participation with professionalism and intellectual integrity.

If I have succeeded here, I have clarified the state of the art of aesthetics and criticism in HCI, and made the case that our field could be improved with more rigorous use of their strategies. It is perhaps fitting, therefore, to conclude by describing some mappings of criticism and HCI (and in doing so I'd like to acknowledge the participants of the critical HCI workshop at British HCI [10]). Criticism can contribute to the practice of $\mathrm{HCI}$ in the following ways:

- Informing the existing design process. Critical theory can inform one or more stages of the existing interaction design process (e.g., user research, prototyping, and evaluation) by enhancing our ability to discover and explore subtle phenomena that we, in turn, might want to build into research hypotheses. [11]'s pastiche scenarios use critical theory to add a rich dimension to a traditional design activity.

- Critical theory can resist or innovate on the design process. The conceptual approaches of [30], for example, often explicitly rely on critical theory to subvert traditional design approaches, to discover new ways to generate new kinds of design insights.

- Interaction design can develop theory. HCI need not passively accept what has already been developed in critical theory. Interaction design is arguably the dominant cultural medium today, and we can innovate on critical theory, to make it work better for our community's professional and intellectual needs, from new design frameworks to educational vocabularies for design educators and professional mentors.

- Interaction criticism can expose the consequences of design. Researchers can critique interaction designs with the hope of exposing unintended consequences and enabling the community to design more rationally. Ontological design, as it evolved into sustainable design, is an example in which critical activity contributed to the emergence of an important new domain of HCI research and practice.

Of these four, the first two remain explicitly in service of design. The latter two serve design indirectly, by contributing to the health of the discipline. In no case do any of these mappings reject or replace the scientific approaches that continue to serve HCI. Rather, inasmuch as aesthetics and critical theory have already become a part of HCI's increasingly cultural agenda, they are worth taking as seriously as we take the rest of our work.

\section{REFERENCES}

1. Agre, P. Computation and Human Experience. Cambridge: Cambridge University Press (1997).

2. Bardzell, J. \& Bardzell, S. Intimate interactions: Online representation and software of the self. Interactions $x v 5$, ACM Press (2008), 11-15.
3. Bardzell, J. Creativity in amateur multimedia: popular culture, critical theory, and HCI. Human Technology 3 (1) (2007), 12-33.

4. Bardzell, J. \& Bardzell, S. Interaction criticism: a proposal and framework for a new discipline of HCI. In CHI '08 Extended Abstracts. ACM, New York, NY, 2463-2472.

5. Barthes, R. The death of the author. In Heath, S. (trans). Image-Music-Text. Hill and Wang, New York (1977), 142-148.

6. Bazin, A. What is Cinema? Volumes 1 and 2. University of California Press, Berkeley and Los Angeles, USA. (1967).

7. Bertselsen, O. \& Pold, S. Criticism as an approach to interface aesthetics. Proc. of NordiCHI'04, ACM Press (2004), 23-32.

8. Blandford, A. Coherence, Community and Strategy in HCI. HCI2007; http://www.future-ukhci.org.uk/ppts/panel_intro.ppt

9. Blevis, E. Sustainable interaction design: invention \& disposal, renewal \& reuse. Proc. of CHI'07, ACM Press (2007), 503-512.

10. Blythe, M., Bardzell, J., Bardzell, S., and Blackwell, A. Critical Issues in Interaction Design. HCI 2008, Culture, Creativity and Interaction Design (2008).

11. Blythe, M. A. and Wright, P. C. 2006. Pastiche scenarios: Fiction as a resource for user centred design. Interact. Comput. 18, 5 (Sep. 2006), 1139-1164.

12. Boehner, K., DePaula, R., Dourish, P., and Sengers, P. Affect: From information to interaction. AARHUS'05, ACM Press (2005), 59-67.

13. Bolter, J, \& Grusin, R. Remediation. MIT Press (1999).

14. Bonanni, L., Parkes, A., and Ishii, H. 2008. Future craft: how digital media is transforming product design. In Proc of CHI '08. ACM, New York, NY, 2553-2564.

15. Buchanon, R. Declaration by design: Rhetoric, argument, and demonstration in design practice. In Margolin, V. (ed.). Design Discourse: History, Theory, Criticism. (1989).

16. Carnap, R. The nature of theories. In Klemke, E.D., Hollinger, R., and Rudge, D.W. (ed). Introductory Readings in the Philosophy of Science. Amherst, NY: Prometheus Books (1998).

17. Coyne, R. Designing Information Technology in the Postmodern Age: From Method to Metaphor. The MIT Press (1997).

18. De Beauvoir, S. The Second Sex. New York: Vintage Books (1989 [1952]).

19. Dickie, G. Introduction to Aesthetics: An Analytic Approach. Oxford UP (1997).

20. Dourish, P., Finlay, J., Sengers, P., \& Wright, P. Reflective HCI: Towards a critical technical practice. In 
CHI'04 Extended Abstracts. ACM Press (2004), 17271728.

21.Eco, U. The Role of the Reader: Explorations in the Semiotics of Texts. Indiana University Press, Bloomington Indian, USA (1979/1984).

22. Fishwick, P. (ed.). Aesthetic Computing. The MIT Press (2006).

23. Fishwick, P. An introduction to aesthetic computing. In Fishwick, P. (ed.) Aesthetic Computing. The MIT Press (2006), 3-28.

24. Fogg, B. J. Persuasive Technology: Using Computers to Change What We Think and Do. Morgan Kaufmann, San Francisco (2003).

25. Foucault, M. The History of Sexuality, Volume 2: The Use of Pleasure. New York: Vintage (1990 [1984]).

26. Fry, T. A New Design Philosophy: An Introduction to Defuturing. New South Wales, Australia: NSWU Press. (1999).

27. Gadamer, H.-G. The Relevance of the Beautiful and Other Essays. Cambridge: Cambridge University Press, 1987.

28. Gajendar, U. Experiential aesthetics: A framework for beautiful experience. Interactions xv 5, ACM Press (2008), 6-10.

29. Gaver, B., Dunne, T., and Pacenti, E. 1999. Design: Cultural probes. Interactions 6, 1 (Jan. 1999), 21-29.

30. Gaver, W. W., Bowers, J., Boucher, A., Gellerson, H., Pennington, S., Schmidt, A., Steed, A., Vikkars, N., and Walker, B. The drift table: Designing for ludic engagement. In CHI'04 Extended Abstract. ACM Press (2004).

31. Graham, G. Philosophy of the Arts: An Introduction to Aesthetics. Routledge (1997).

32. Greenberg, S., and Buxton, B. Usability evaluation considered harmful (some of the time). Proc. of CHI'08, ACM Press (2008), 111-120.

33. Hammermeister, K. The German Aesthetic Tradition. Cambridge: Cambridge University Books (1998).

34. Hanks, K., Odom, W., Roedl, D. and Blevis, E. Sustainable millennials: Attitudes towards sustainability and the material effects of interactive technologies. Proc. of CHI'08, ACM Press (2008), 333-342.

35. Hayles, N. K. How We Became Posthuman: Virtual Bodies in Cybernetics, Literature, and Informatics. University of Chicago Press (1998).

36. Kivy, P. (ed.). The Blackwell Guide to Aesthetics. Blackwell Publishing (2004).

37. McCarthy, J. and Wright, P. Technology as Experience. The MIT Press (2004).

38. McLaren, M. Feminism, Foucault, and Embodied Subjectivity. State University of New York Press (2002).
39. Landow, G. Hypertext 2.0: The Convergence of Contemporary Critical Theory and Technology. The Johns Hopkins UP (1997).

40.Levinson, J. (ed.). The Oxford Handbook of Aesthetics. Oxford UP (2003).

41.Löwgren, J. \& Stolterman, E. Thoughtful Interaction Design. MIT Press (2004).

42. Manovich, L. The Language of New Media. MIT Press (2001).

43. Nelson, H., and Stolterman, E. The Design Way. Educational Technology Publications (2002).

44. Nielsen, J. Ten usability heuristics. Online: www. useit.com/papers/heuristic/heuristic_list.html

45. Norman, D. The Design of Everyday Things. Basic Books, New York (1988).

46. Perer, A. and Shneiderman, B. 2008. Integrating statistics and visualization: Case studies of gaining clarity during exploratory data analysis. In Proc of CHI'08. ACM, New York, NY, 265-274.

47.Picard, R. Affective Computing. MIT Press, Cambridge, MA, USA, 1997.

48. Rehm, M., André, E., Nakano, Y., Nishida, T. Enculturating $\mathrm{HCI}$ (http://mm-werkstatt.informatik.uniaugsburg.de/documents/EHCI/)

49. Sengers, P., Boehner, K., David, S. \& Kaye, J. Reflective design. CC '05. ACM Press, New York, NY, 49-58.

50. Sengers, P. and Gaver, B. Staying open to interpretation: Engaging multiple meanings in design and evaluation. Proc. of DIS 2006, ACM Press (2006), 899-108.

51. Sengers, P., McCarthy, J. \& Dourish, P. (2006). Reflective HCI: articulating an agenda for critical practice. Extended Abstracts, CHI '06, ACM Press, New York, NY, 1683-1686.

52. Sharp, H., Rogers, Y., and Preece, J. Interaction Design. John Wiley \& Sons, Ltd. (2007).

53. Shusterman, R. Pragmatist Aesthetics: Living Beauty, Rethinking Art. Lanham, MD: Rowman \& Littlefield Publishers (2000).

54. Stroll, A. Twentieth-Century Analytic Philosophy. Columbia UP (2000).

55. Tompkins, J. (ed.). Reader-Response Criticism: From Formalism to Post-Structuralism. The Johns Hopins University Press: Baltimore USA (1980).

56. Udsen, L, and Jørgensen, A. The Aesthetic Turn. Digital Creativity, 16 (4), 205-216.

57. Willis, A.M. (2006). Ontological designing. Design Philosophy Papers. \#02/2006.

58. Winograd, T. and Flores, W. Understanding Computers and Cognition. Addison-Wesley, Norwood, NJ. (1986) 